\title{
A rare case of pediatric Crohn's disease and alveolar bone loss: a report and review
}

\author{
Mei-Li Huang ${ }^{1 \#}$, Ying-Qian $W^{2,3 \#}$, Wen-Hua Ruan ${ }^{2,3}$ \\ ${ }^{1}$ Department of Otolaryngology Head and Neck Surgery, Sir Run Run Shaw Hospital, Zhejiang University School of Medicine, Hangzhou, China; \\ ${ }^{2}$ Department of Stomatology, the Children's Hospital, Zhejiang University School of Medicine, National Clinic Research Center for Child Health, \\ Hangzhou, China; ${ }^{3}$ Key Laboratory of Oral Biomedical Research of Zhejiang Province, Zhejiang University School of Stomatology, Hangzhou, \\ China \\ \#These authors contributed equally to this work. \\ Correspondence to: Wen-Hua Ruan. Department of Stomatology, the Children's Hospital, Zhejiang University School of Medicine, National Clinical \\ Research Center for Child Health, Hangzhou 310052, China; Key Laboratory of Oral Biomedical Research of Zhejiang Province, Zhejiang \\ University School of Stomatology, Hangzhou 310003, China. Email: zyeykqk@zju.edu.cn.
}

\begin{abstract}
Crohn's disease (CD) is a granulomatous inflammatory bowel disease (IBD) that can affect any part of the gastrointestinal tract, but its etiology is still unclear. CD patients usually have extraintestinal manifestations including oral manifestations, and this can also occasionally appear in children. Oral manifestations, including aphthous stomatitis, typically develop on the mucocutaneous tissue. As pediatric $\mathrm{CD}$ is not common, there have been no reports concerning alveolar bone resorption for these kinds of patients. An 11-year-old Chinese girl was referred to our hospital because of reoccurring painful oral ulcers, with a 2.5-year history of $\mathrm{CD}$ and recurrent oral ulcers. Clinical examinations revealed intestinal lesions, oral ulcers, and alveolar bone loss. CD and its related periodontitis were diagnosed. After comprehensive therapy, including periodontal scaling and anti-inflammatory treatment, the oral ulcers gradually healed. One year later, follow-up visit showed the anterior alveolar bone to be stable and new alveolar bone regenerating around the mandibular anterior teeth at some sites. It is rare for CD children to have periodontitis, especially with severe alveolar bone loss. This case suggests that when treating periodontitis in children, the possibility of systemic disease, such as CD, should be considered and attended to. Early diagnosis and symptomatic treatment and care are crucial.
\end{abstract}

Keywords: Crohn's disease (CD); children; alveolar bone; extraintestinal involvement; case report

Submitted Aug 04, 2020. Accepted for publication Sep 27, 2020.

doi: $10.21037 /$ tp-20-279

View this article at: http://dx.doi.org/10.21037/tp-20-279

\section{Introduction}

Crohn's disease $(\mathrm{CD})$ is a chronic inflammatory disorder that can affect any part of the gastrointestinal tract. Patients often suffer from persistent diarrhea, abdominal pain, and weight loss. The patients usually have extraintestinal manifestations, and $5.8 \%$ of them have oral manifestations, including aphthous stomatitis, pyostomatitis vegetans, indurated tag-like lesions, and periodontal inflammation (1). In more recent study, Zhang and his colleagues found that CD adults had significantly higher percentages of sites with probing pocket depth $\geq 5 \mathrm{~mm}$ when compared with controls (2). Nevertheless, pediatric $\mathrm{CD}$ is rare and, to our knowledge, no reports on CD children suffering from alveolar bone destruction have been published.

Here, we present a case of a young girl who had suffered from CD for many years, and who was revealed to have severe alveolar bone destruction.

We present the following article in accordance with the CARE reporting checklist (available at http://dx.doi. org/10.21037/tp-20-279). 

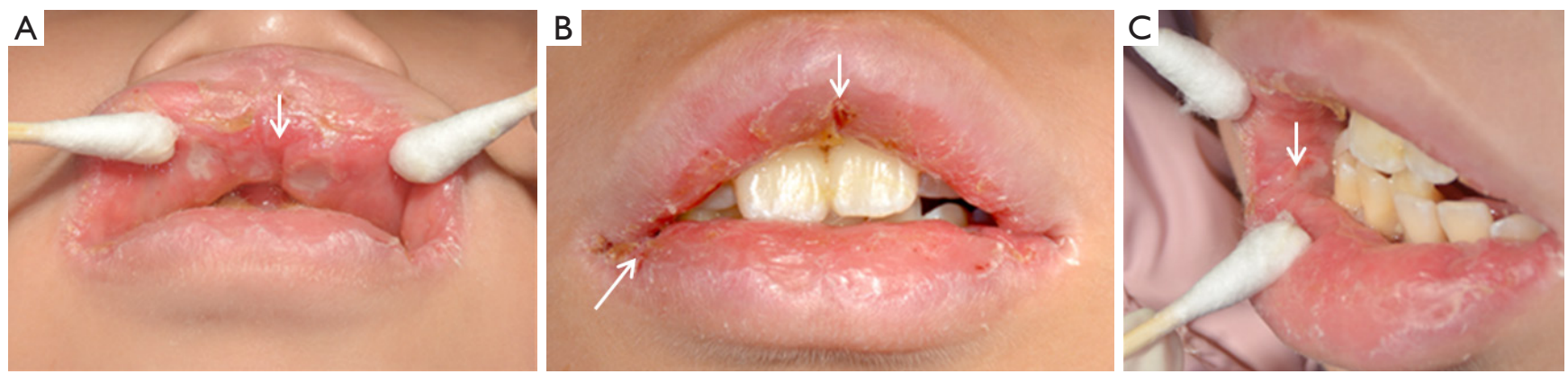

Figure 1 Upper and lower swollen lips with distinct vertical fissures (as indicated by arrows).

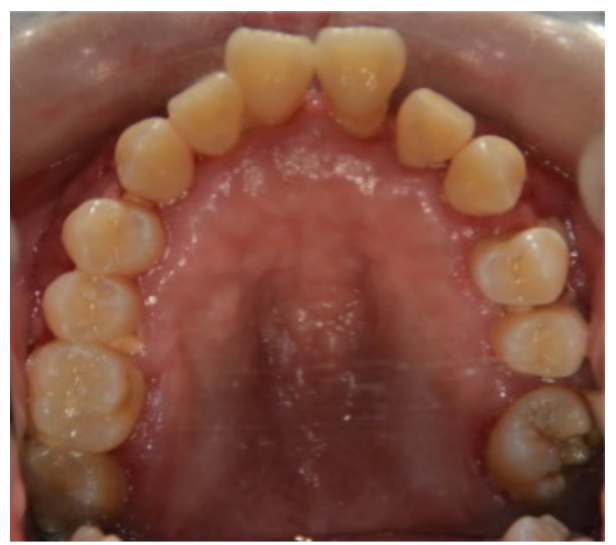

Figure 2 Cobblestoning of the mucosa throughout the palate.

\section{Case presentation}

A Chinese girl, 11 years and 11 months old, who was initially diagnosed as CD 2.5 years ago, was referred to the Children's Hospital, Zhejiang University School of Medicine due to recurrent oral painful ulcers. She had no family history of inflammatory bowel disease (IBD).

Due to abdominal pain, bloody stool, and recurrent oral ulceration, she was hospitalized for a comprehensive examination 2.5 years prior. Magnetic resonance imaging (MRI) of the small intestine exhibited segmental thickening of the intestinal wall of the ascending colon, descending colon, and sigmoid colon, which confirmed the diagnosis of CD. After taking metronidazole, infliximab, Etiasa, 6-mercaptopurine, and parenteral nutrition, she recovered and was discharged. After this, she periodically experiences abdominal pain, diarrhea, bloody stool, and aphthous mucosal ulcers. These symptoms cleared after she took the abovementioned medicine. However, she found that the symptoms were worsening, and the some of her teeth had become loose.
One week prior to her visit, she discovered oral ulcers again, accompanied by red and swollen lips, and a fever with a peak temperature of $37.8{ }^{\circ} \mathrm{C}$. The painful ulcers were scattered throughout her oral cavity. Meanwhile, her anterior teeth had become looser than before. After being treated in the gastroenterology department, she was referred to the dental and otorhinolaryngology clinic. There was no obvious otorhinolaryngological finding, while dental inspection revealed that the girl had swollen lips with vertical fissures (Figure 1). Mucosa involvements included dispersed irregular ulcers, cobblestone palate (Figure 2), and buccal tag-like lesions. Dental examination indicated that tooth 16 was missing and teeth 17,36 , and 46 had large decayed cavities on the occlusal surface. Calculus and debris had accumulated around the root surface of the whole teeth. Almost all areas of the gingiva were congestive and severely recessed, which resulted in the roots being observably exposed (Figure 2). The mobility of the 8 anterior teeth was grade III, while teeth 11 and 41 had been elongated. Her Ramfjord plaque index was 3.17 while her gingival index was 1.83 . Due to the unbearable pain originating from the mucosal ulcers, the girl had great difficulty opening her mouth, and so we could not take periapical radiographs. Orthopantomography was performed, which indicated that there was alveolar bone loss of the anterior teeth (Figure 3). Therefore, the girl was diagnosed with CD and periodontitis.

Comprehensive measures were taken, including oral health education, chlorhexidine mouthwash, simple periodontal scaling, intravenous piperacillin sodium and tazobactam sodium, and metronidazole and folic acid prescription. About one 1 later, her swollen lips, mucosal ulcerations, and congestive gingival were alleviated and she could open her mouth wide. Therefore, standard periodontal scaling was applied to promote the recovery of periodontal tissue. Meanwhile, periapical X-rays of the 


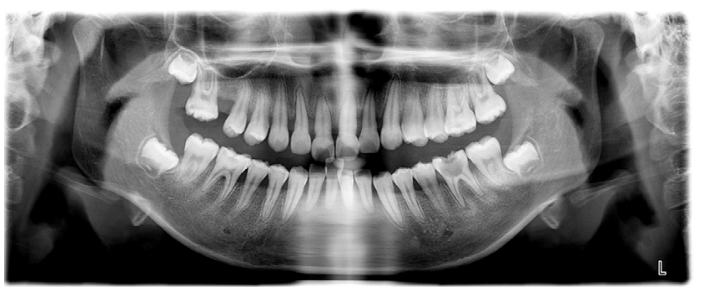

Figure 3 Orthopantomography displaying alveolar bone loss in the areas of the tooth $11,12,21,22$, and 46 .

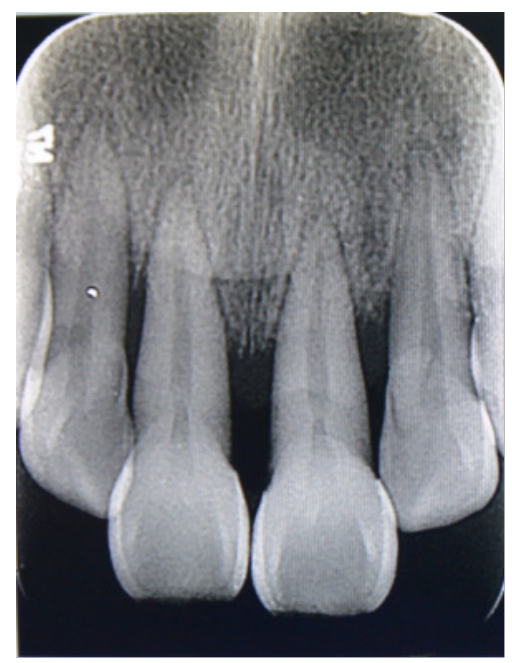

Figure 4 A X-ray film showing upper anterior alveolar bone loss.

anterior teeth were taken, which revealed severe alveolar bone resorption both in the upper and lower anterior areas. Alveolar bone loss in tooth 41 had almost even reached the apical area (Figures 4,5).

One year later, a follow-up visit revealed that her oral hygiene had improved along with the mobility of her the anterior teeth. Her Ramfjord plaque index decreased to 2.17 while her gingival index reduced to 1.50 . Also, the mobility of the 8 anterior teeth was almost II. Radiographs revealed that the anterior alveolar bone was stable, and in some sites, new alveolar bone was regenerating around the mandibular anterior teeth (Figures 6,7).

The outcome pleased the girl and encouraged her to be more active in her oral hygiene.

All procedures performed in this study involving human participants were in accordance with the Declaration of Helsinki (as revised in 2013). Written informed consent was obtained from the patient for publication of this study and any accompanying images.

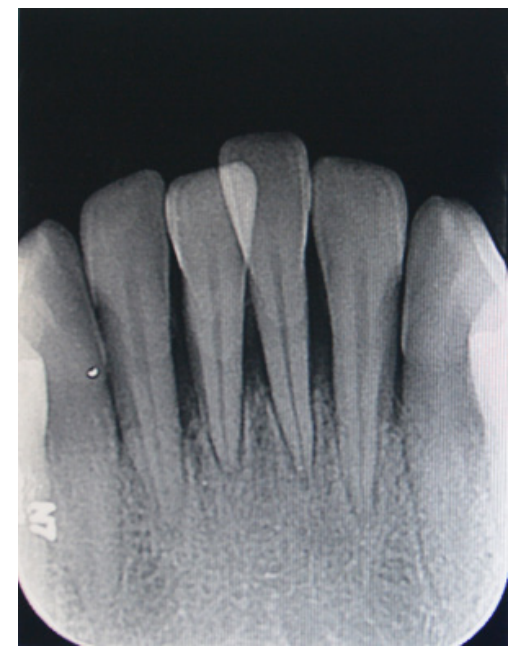

Figure 5 A X-ray film showing serious lower anterior alveolar bone loss.

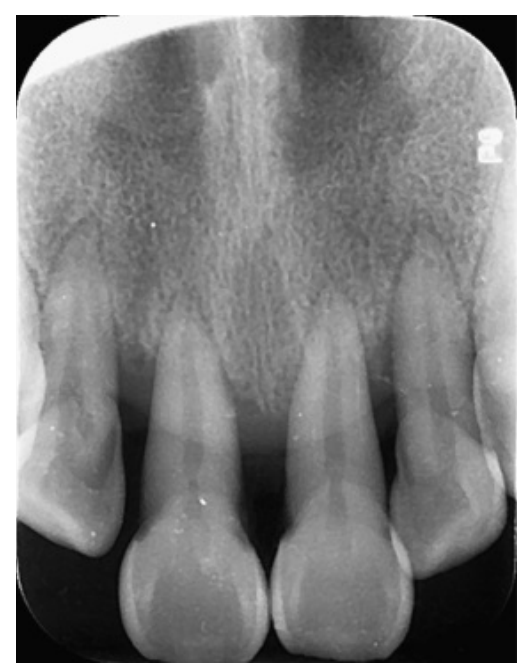

Figure 6 Periapical film revealed that the alveolar bone had remained stable one year after comprehensive treatment

\section{Discussion}

The young CD girl displayed specific oral manifestations $(3,4)$, including cobblestone mucosa, mucosal tags, recurrent aphthous ulcers, and swollen lips with vertical fissures. She was also afflicted with severe periodontal destruction, including gingival recession and alveolar bone resorption. According to our knowledge of the existing literature, this is the first report to describe alveolar loss in CD children.

Investigations have discovered that $\mathrm{CD}$ adults often have soft tissue problems in the oral cavity, which can include 


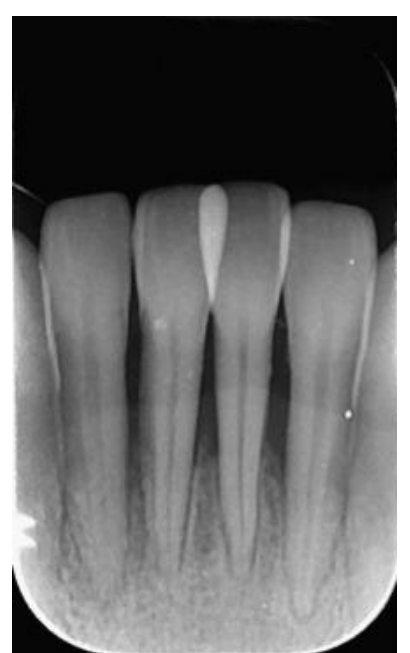

Figure 7 X-ray film showed the alveolar bone had recovered and that new regenerating bone was forming in some sites around the mandibular anterior teeth.

oral ulcerations, cobblestone mucosa, and granulomatous cheilitis (5-7). These patients may also experience concurrent periodontal involvements, including higher tendency of bleeding on probing, deeper periodontal pocketing, and significant alveolar bone loss when compared to the general population (8-11). The hazard ratio for subsequent periodontitis among patients with $\mathrm{CD}$ was found to be 1.36 compared to healthy subjects (11).

It is widely acknowledged that CD children have a few specific features that are different from CD adults. The prevalence of oral manifestations in IBD has been reported to range widely from $7.3 \%$ to $41 \%$ in children $(3,12)$. Most oral manifestations are mucocutaneous findings $(3,8)$, and oral mucosal lesions can be specific or nonspecific. Among the specific lesions correlated to $\mathrm{CD}$, are mucosal tags, cobblestone mucosa, and labial swelling linked to granulomatous cheilitis. One prospective 3-year study showed that more than one-third of all children presenting with CD had involvement of the mouth. The oral findings included mucogingivitis, mucosal tags, deep ulceration, cobblestoning, lip swelling, and pyostomatitis vegetans (3). Typical mucosal lesions were found in our patient.

Many reports have indicated a higher prevalence of dental caries and periodontal disease in adults with IBD $(13,14)$. Recently, some investigators found this adult phenomenon could also be present in CD children (15). A Greek case control study by Koutsochristou et al. (15) reported that IBD children had a statistically significant higher index of decayed, missing, and filled teeth (dmf-t or DMF-T) compared to controls, while the community periodontal index of treatment needs (CPITN) was significantly higher compared with that of controls. In our case, the girl's dental caries and periodontal health were serious. The increase in dental caries risk for CD patients was thought to be associated with dietary habits, changes in salivary and microbiological conditions of the oral cavity, and malabsorption of vitamin D (16). Both caries and periodontitis appear to result from decreased biodiversity and local microbial community perturbation $(4,17,18)$. Although Koutsochristou et al. (15) applied the CPITN index to evaluate periodontal treatment needs of children with IBD and found that $9 \%$ had a CPITN score of 3 (at least 1 site with probing pocket depth value between 4 and $5 \mathrm{~mm}$ ), they did not reveal any deep alveolar bone loss while probing for pocket depth. On occasion, a deep probing pocket does imply alveolar bone loss. A 2004 case report described a 6-year-old CD boy who had an increased periodontal probing depth but no visible alveolar loss on radiography (19). In our case, the girl presented with periodontal manifestations including serious alveolar resorption which was radiographically visible.

According to the World Workshop on the Classification of Periodontal and Peri-Implant Diseases and Conditions, which was co-sponsored by the American Academy of Periodontology and the European Federation of Periodontology (2017), a new approach for periodontitis staging, based on four stages and three grades instead of chronic and aggressive periodontitis, was explained. Therefore, due to systemic involvements, CD belongs to manifestations of systemic diseases and conditions that affect the periodontal attachment apparatus. This consensus report asserted that IBD shows the similar immunopathogenic responses to periodontitis and that there exists a significant correlation between these two diseases (20). Further evidence suggests that CD in the oral cavity may precede gastrointestinal involvement and that even around $30 \%$ of CD cases in children arise first in the mouth $(21,22)$. We thus need to examine any oral involvements, including the integrity of the mucous membrane and periodontal condition, as early as possible so that further testing for $\mathrm{CD}$ can be initiated.

There have been many studies on the mechanism by which IBD induces periodontitis $(19,23,24)$. LiraJunior and Figueredo (23) speculated that there exists an interaction between IBD and periodontitis. IBD probably induces periodontitis via changing the oral microbiota and 
the inflammatory response. The induction of periodontitis seems to result in gut dysbiosis and altered gut epithelial cell barrier function, which might contribute to the pathogenesis of IBD. Moreover, some scholars have reported higher levels of circulating immune complex activity and greater polymorphonuclear leukocyte metabolic activity in active IBD patients, and surmised that these activities are related to rapidly progressive alveolar bone loss (24). In addition, the perturbation of the cytokine network (25), changes in salivary function (26), and colonization of Actinobacillus actinomycetemcomitans may also be related to the destruction of the alveolar bone in CD children (19).

Currently, 6-mercaptopurine and other thiopurines are still widely used in patients with IBD $(27,28)$. However, it is worth noting that the long-term use of these medications may change periodontal status and provoke gingival overgrowth. The mechanism of gingival hyperplasia is still being debated and individual sensitivity, age, or dose and duration of the drug therapy may be related to this gingival enlargement $(15,29)$. Mouth rinse and oral hygiene instruction for these patients are recommended.

\section{Conclusions}

As pediatric CD may result in alveolar bone loss at an early age, early complete oral examination and periodic maintenance, together with inclusion of a gastroenterologist for systemic therapy, are essential in facilitating early diagnosis and can improve patients' quality of life. As dentists, we must be aware of oral CD involvements, including swollen lips with fissure, linear mucosal ulceration, cobblestone mucosa, and tag-like mucosal lesions. Oral manifestations may precede or co-occur with intestine symptoms, and comprehensive measures should be taken for this kind of patient.

\section{Acknowledgments}

Funding: None.

\section{Footnote}

Reporting Checklist: The authors have completed the CARE reporting checklist. Available at http://dx.doi.org/10.21037/ tp-20-279

Conflicts of Interest: All authors have completed the ICMJE uniform disclosure form (available at http://dx.doi. org/10.21037/tp-20-279). The authors have no conflicts of interest to declare.

Ethical Statement: The authors are accountable for all aspects of the work in ensuring that questions related to the accuracy or integrity of any part of the work are appropriately investigated and resolved. All procedures performed in this study involving human participants were in accordance with the Declaration of Helsinki (as revised in 2013). Written informed consent was obtained from the patient for publication of this study and any accompanying images.

Open Access Statement: This is an Open Access article distributed in accordance with the Creative Commons Attribution-NonCommercial-NoDerivs 4.0 International License (CC BY-NC-ND 4.0), which permits the noncommercial replication and distribution of the article with the strict proviso that no changes or edits are made and the original work is properly cited (including links to both the formal publication through the relevant DOI and the license). See: https://creativecommons.org/licenses/by-nc-nd/4.0/.

\section{References}

1. Löwenberg M. Is there a beneficial effect of adding azathioprine to adalimumab in Crohn's disease patients? Ann Transl Med 2018;6:278.

2. Gjuladin-Hellon T, Iheozor-Ejiofor Z, Gordon M, et al. Azathioprine and 6-mercaptopurine for maintenance of surgically-induced remission in Crohn's disease. Cochrane Database Syst Rev 2019;8:CD010233.

3. Bermejo F, Aguas M, Chaparro M, et al. Recommendations of the Spanish Working Group on Crohn's Disease and Ulcerative Colitis (GETECCU) on the use of thiopurines in inflammatory bowel disease. Gastroenterol Hepatol 2018;41:205-21.

4. de Vries SAG, Tan CXW, Bouma G, et al. Salivary Function and Oral Health Problems in Crohn's Disease Patients. Inflamm Bowel Dis 2018;24:1361-7.

5. Brandtzaeg P. Inflammatory bowel disease: clinics and pathology. Do inflammatory bowel disease and periodontal disease have similar immunopathogeneses? Acta Odontol Scand 2001;59:235-43.

6. Lamster IB, Rodrick ML, Sonis ST, et al. An analysis of peripheral blood and salivary polymorphonuclear leukocyte function, circulating immune complex levels and oral status in patients with inflammatory bowel disease. J 
Periodontol 1982;53:231-8.

7. Lira-Junior R, Figueredo CM. Periodontal and inflammatory bowel diseases: Is there evidence of complex pathogenic interactions? World J Gastroenterol 2016;22:7963-72.

8. Eckel A, Lee D, Deutsch G, et al. Oral manifestations as the first presenting sign of Crohn's disease in a pediatric patient. J Clin Exp Dent 2017;9:e934-8.

9. Woo VL. Oral Manifestations of Crohn's Disease: A Case Report and Review of the Literature. Case Rep Dent 2015;2015:830472.

10. Albandar JM, Susin C, Hughes FJ. Manifestations of systemic diseases and conditions that affect the periodontal attachment apparatus: Case definitions and diagnostic considerations. J Periodontol 2018;89 Suppl 1:S183-S203.

11. Sigusch BW. Periodontitis as manifestation of Crohn's disease in primary dentition: a case report. J Dent Child (Chic) 2004;71:193-6.

12. Gao L, Xu T, Huang G, et al. Oral microbiomes: more and more importance in oral cavity and whole body. Protein Cell 2018;9:488-500.

13. Costalonga M, Herzberg MC. The oral microbiome and the immunobiology of periodontal disease and caries. Immunol Lett 2014;162:22-38.

14. Kabbani TA, Koutroubakis IE, Schoen RE, et al. Association of Vitamin D Level With Clinical Status in Inflammatory Bowel Disease: A 5-Year Longitudinal Study. Am J Gastroenterol 2016;111:712-9.

15. Koutsochristou V, Zellos A, Dimakou K, et al. Dental Caries and Periodontal Disease in Children and Adolescents with Inflammatory Bowel Disease: A CaseControl Study. Inflamm Bowel Dis 2015;21:1839-46.

16. Bevenius J. Caries risk in patients with Crohn's disease: a pilot study. Oral Surg Oral Med Oral Pathol 1988;65:304-7.

17. Szymanska S, Lördal M, Rathnayake N, et al. Dental caries, prevalence and risk factors in patients with Crohn's disease. PLoS One 2014;9:e91059.

18. Greuter T, Bertoldo F, Rechner R, et al. Extraintestinal Manifestations of Pediatric Inflammatory Bowel Disease: Prevalence, Presentation, and Anti-TNF Treatment. J Pediatr Gastroenterol Nutr 2017;65:200-6.

Cite this article as: Huang ML, Wu YQ, Ruan WH. A rare case of pediatric Crohn's disease and alveolar bone loss: a report and review. Transl Pediatr 2020;9(5):720-725. doi: 10.21037/tp20-279
19. Chi YC, Chen JL, Wang LH, et al. Increased risk of periodontitis among patients with Crohn's disease: a population-based matched-cohort study. Int J Colorectal Dis 2018;33:1437-44.

20. Yu HC, Chen TP, Chang YC. Inflammatory bowel disease as a risk factor for periodontitis under Taiwanese National Health Insurance Research database. J Dent Sci 2018;13:242-7.

21. Brito F, de Barros FC, Zaltman C, et al. Prevalence of periodontitis and DMFT index in patients with Crohn's disease and ulcerative colitis. J Clin Periodontol 2008;35:555-60.

22. Haaramo A, Alapulli H, Aine L, et al. Oral and Otorhinolaryngological Findings in Adults Who Were Diagnosed With Pediatric Onset Crohn's Disease: A Controlled Study. J Clin Gastroenterol 2019;53:e269-75.

23. Habashneh RA, Khader YS, Alhumouz MK, et al. The association between inflammatory bowel disease and periodontitis among Jordanians: a case-control study. J Periodontal Res 2012;47:293-8.

24. Muhvić-Urek M, Tomac-Stojmenović M, MijandrušićSinčić B. Oral pathology in inflammatory bowel disease. World J Gastroenterol 2016;22:5655-67.

25. Tan CX, Brand HS, de Boer NK, et al. Gastrointestinal diseases and their oro-dental manifestations: Part 1: Crohn's disease. Br Dent J 2016;221:794-9.

26. Lauritano D, Boccalari E, Di Stasio D, et al. Prevalence of Oral Lesions and Correlation with Intestinal Symptoms of Inflammatory Bowel Disease: A Systematic Review. Diagnostics (Basel) 2019;9:77.

27. Li GW, Ren JA. Pay attention to the study of Crohn's disease epidemiology in China. Parenteral \& Enteral Nutrition 2017;24:135-7.

28. Harty S, Fleming P, Rowland M, et al. A prospective study of the oral manifestations of Crohn's disease. Clin Gastroenterol Hepatol 2005;3:886-91.

29. Zhang L, Gao X, Zhou J, et al. Increased risks of dental caries and periodontal disease in Chinese patients with inflammatory bowel disease. Int Dent J 2020;70:227-36.

(English Language Editor: J. Gray) 